\title{
Análise do tempo de ventilação mecânica e internamento em pacientes submetidos a cirurgia cardíaca
}

\author{
Analysis of mechanical ventilation time and hospitalization of patients \\ undergoing cardiac surgery
}

André Luiz Lisboa Cordeiro ${ }^{1}$, Adrianna Souza Silva de Lima ${ }^{1}$, Isis Caroline Oliveira Matos ${ }^{1}$, Lais Vilas Boas Oliveira ${ }^{1}$, André Raimundo Guimarães², Sarah de Oliveira Carvalho², Thiago Araújo de Melo $^{3}$

${ }^{1}$ Faculdade Nobre (FAN) - Feira de Santana (BA), Brasil.

2Instituto Nobre de Cardiologia (INCARDIO), Santa Casa de Misericórdia - Feira de Santana (BA), Brasil.

3Universidade Salvador (UNIFACS) - Salvador (BA), Brasil.

DOI: http://dx.doi.org/10.7322/abcshs.v42i1.942

\section{RESUMO}

Introdução: Pacientes submetidos à cirurgia cardíaca (CC) necessitam de ventilação mecânica invasiva (VMI) após o procedimento cirúrgico, sendo que um tempo prolongado de VMI pode levar a um aumento do tempo de permanência na Unidade de Terapia Intensiva (UTI). Objetivo: Verificar se o tempo de VMI interfere no tempo de internação em UTI. Métodos: Foram analisados, prospectivamente, pacientes submetidos à CC e que permaneceram por um tempo inferior a 24 horas na VMI. Foram selecionados 69 pacientes, os quais foram admitidos na UTI e acompanhados até o momento da extubação. Com esse dado encontrou-se o tempo que permaneceram em VMI e, depois, quantos dias permaneceram internados na UTI até o momento da alta da unidade. Resultados: A maior parte dos pacientes submetidos à CC foi do sexo masculino (56,5\%); a idade média foi de 55,93 $\pm 14,29$ anos; o tipo de cirurgia mais prevalente foi a revascularização miocárdica (71,0\%); o tempo médio de circulação extracorpórea (CEC) foi de 65,05 $\pm 20,06$ minutos; o tempo médio de internação na UTI foi de 2,18+1,10 dias; o tempo de VMI foi de 7,32 $\pm 2,66$ horas. Não se verificou significância estatística entre essas duas últimas variáveis com um $p=0,43$. Conclusão: Pode-se concluir que não houve correlação entre o tempo de ventilação mecânica e tempo de internação em UTI em pacientes que não apresentaram complicações pós-operatórias imediatas e que permaneceram menos de 12 horas sob ventilação mecânica.

Palavras-chave: respiração artificial; cirurgia torácica; unidades de terapia intensiva.

\section{ABSTRACT}

Introduction: Patients undergoing cardiac surgery (CS) require invasive mechanical ventilation (IMV) after surgery and a prolonged IMV can lead to an increased length of stay in the Intensive Care Unit (ICU). Objective: To determine if the length of IMV interferes in ICU length of stay. Methods: Patients undergoing CS and that remained less than 24 hours on IMV were prospectively analyzed. A total of 69 selected patients were admitted to the ICU and followed until extubation. With this information, it was possible to know the time that they remained in IMV and then how many days they stayed in the ICU until the time of discharge from the unit. Results: Most patients undergoing CS were male (56.5\%); the mean age was $55.93 \pm 14.29$ years; the most prevalent type of surgery was myocardial revascularization $(71.0 \%)$; the average time of cardiopulmonary bypass (CPB) was $65.05 \pm 20.06$ minutes; the average length of stay in ICU was $2.18 \pm 1.10$ days; the IMV time was $7.32 \pm 2.66$ hours. There was no statistical significance between these two last variables with $p=0.43$. Conclusion: It can be concluded that there was no correlation between the duration of mechanical ventilation and ICU length of stay in patients without immediate postoperative complications and who spent less than 12 hours of mechanical ventilation.

Keywords: respiration, artificial; thoracic surgery; intensive care units. 


\section{INTRODUÇÃO}

A doença cardíaca é uma das doenças que apresenta maior mortalidade mundialmente. No Brasil, a taxa de mortalidade no que diz respeito ao Infarto Agudo do Miocárdio (IAM) e Insuficiência Cardíaca (IC), em caráter de urgência, foi de 10,20\% no mês de janeiro de 2014, segundo o Departamento de Informática do Sistema Único de Saúde do Brasil (DATASUS) ${ }^{1}$. Essa mesma fonte relata que foram realizadas 10.652 cirurgias de revascularização do miocárdio e/ou troca valvar durante o período de janeiro a junho de 2008 em todo o Brasil ${ }^{1}$. Mesmo com todo o avanço tecnológico que visa aumentar e prolongar a qualidade vida dos pacientes submetidos a essa cirurgia, esse procedimento continua sendo de alta complexidade e favorece alterações nas funções pulmonar e periférica ${ }^{2}$.

O paciente no pós-operatório de cirurgia cardíaca necessita da ventilação mecânica invasiva (VMI) durante algumas horas. Segundo Butler et al. ${ }^{3}$, esses pacientes devem ser extubados logo após cessado o efeito da anestesia, sendo que o modo ventilatório não tem impacto sobre a decisão da extubação. Existe uma proposta de extubação traqueal (programa de extubação rápida) em até oito horas e alguns autores relatam a possibilidade desse procedimento ainda no centro cirúrgico, porém o risco de hipotermia, sangramento e instabilidade hemodinâmica são superiores aos benefícios (redução do tempo de internamento hospitalar e na unidade de terapia intensiva (UTI), e complicações respiratórias como atelectasias e derrame pleural) ${ }^{3,4}$. Esse protocolo depende de uma abordagem minimamente invasiva, anestesia baseada em evidências e analgesias 5 .

Alguns fatores estão associados ao prolongamento do tempo de VMI, tais como idade avançada, sexo feminino, tempo de circulação extracorpórea, disfunção cardíaca e baixo débito cardíaco ${ }^{6}$.

Esse processo de extubação precoce está relacionado a uma recuperação mais acelerada da função gastrointestinal, à redução das complicações pulmonares pós-operatórias e à diminuição do tempo de permanência na UTI e no hospital ${ }^{5}$.

Gouvas et al. ${ }^{7}$, em uma meta-análise na qual avaliaram quatro testes controlados randomizados e sete estudos de caso-controle, perceberam que houve uma redução do tempo de estadia hospitalar, da frequência de readmissões e da mortalidade quando aplicado um programa de extubação traqueal rápida.

Portanto, o objetivo deste trabalho foi avaliar se existe impacto do tempo de ventilação mecânica sobre o tempo de internamento hospitalar em pacientes no pós-operatório de cirurgia cardíaca.

\section{MÉTODOS}

De outubro de 2014 a abril de 2015, 69 pacientes foram admitidos na UTI em ventilação mecânica no pós-operatório de cirurgia cardíaca no Instituto Nobre de Cardiologia da Santa Casa de Misericórdia em Feira de Santana, Bahia, os quais foram prospectivamente e consecutivamente incluídos na pesquisa. O estudo atendeu às normas de pesquisa em seres humanos, sendo submetido e aprovado pelo Comitê de Ética em Pesquisa da Faculdade Nobre, em Feira de Santana, Bahia, com o número de aprovação 853.947.

Os critérios de inclusão no estudo foram pacientes admitidos na UTI em VMI no pós-operatório de cirurgia cardíaca, maiores de 18 anos, de ambos os gêneros, e aptos a serem submetidos à interrupção do suporte ventilatório após o despertar. No estudo, foram excluídos: pacientes que permaneceram em VMI por um tempo superior a 24 horas; pacientes com instabilidade hemodinâmica ou qualquer fator que prolongasse o tempo da interrupção da ventilação mecânica (VMI); e pacientes que não aceitaram participar da pesquisa, não assinando o termo de consentimento livre e esclarecido.

Todos os pacientes foram avaliados em um primeiro momento visando ao levantamento de dados epidemiológicos, como idade, gênero e análise dos critérios de inclusão no estudo. Após o procedimento cirúrgico, o paciente era encaminhado para a UTI em ventilação manual e conectado ao ventilador mecânico nessa unidade. Esse era o ponto inicial para contagem do tempo que o mesmo permaneceria em VMI. A avaliação do tempo de VMI era finalizada no momento em que o paciente era extubado e assumia a respiração espontânea, sendo contabilizado esse tempo em horas. Deve-se ressaltar que nenhum dos pesquisadores influenciou o tempo ou a maneira da interrupção do suporte ventilatório, ficando isso a cargo dos profissionais (médico, fisioterapeuta e enfermeiro) envolvidos no cuidado pós-operatório desse paciente. O momento final da pesquisa se deu quando o paciente recebia alta na UTI. Foram contados os dias desde a chegada até a liberação desse paciente da unidade. Mais uma vez, nenhum pesquisador teve influência na decisão de alta bem como na realização de procedimentos que pudessem interferir na liberação do paciente.

\section{RESULTADOS}

Entre os 69 pacientes inclusos no trabalho, 39 (56,6\%) foram homens e 30 (43,5\%) mulheres; a idade média foi de 55,93 $\pm 14,29$ anos. Todos foram submetidos à cirurgia cardíaca, nomeadamente: revascularização miocárdica, 49 (71,0\%); troca de válvula mitral, 16 (23,2\%); correção de comunicação interatrial, 2 (2,9\%); e troca de válvula aórtica, 2 (2,9\%). A Tabela 1 demonstra outras variáveis epidemiológicas e cirúrgicas analisadas.

Como a distribuição dos dados analisados não apresentou padrão de distribuição normal, foi utilizado o teste não paramétrico de Spearman para verificação da significância do ponto de vista estatístico entre as possíveis correlações.

O tempo médio de circulação extracorpórea (CEC) dos pacientes foi de $65,05 \pm 20,06$ minutos, com o tempo médio de VMI de 7,32 $\pm 2,66$ horas, e tempo médio de internação na UTI 2,18 $\pm 1,10$ dias. A análise de correlação entre o tempo de CEC e o tempo de suporte ventilatório invasivo não evidenciou significância do ponto de vista estatístico $(\mathrm{p}=0,23)$. 
Concomitantemente, ao verificar a existência de correlação entre o tempo de VMI e o tempo de internação na UTI (Figura 1), observou-se ausência de significância estatística $(\mathrm{p}=0,43)$. O teste do $\chi^{2}$ também não evidenciou correlação estatística entre o tempo de internação na UTI e o tipo de cirurgia (Figura 2).

\section{DISCUSSÃO}

A frequência dos procedimentos cirúrgicos aumentou progressivamente nas últimas décadas, sendo as complicações

Tabela 1: Características da população do estudo

\begin{tabular}{|c|c|}
\hline Variável & Frequência em \% (n) \\
\hline \multicolumn{2}{|l|}{ Sexo } \\
\hline Masculino & $56,5(39)$ \\
\hline Feminino & $43,5(30)$ \\
\hline \multicolumn{2}{|l|}{ Faixa Etária (anos) } \\
\hline $20-35$ & $10,1(7)$ \\
\hline $36-50$ & $17,4(12)$ \\
\hline $51-65$ & $44,9(31)$ \\
\hline $66-90$ & $27,5(19)$ \\
\hline \multicolumn{2}{|l|}{ Tipo de Cirurgia } \\
\hline Revascularização do Miocárdio (RM) & $71,0(49)$ \\
\hline Troca de Valva Mitral (TVM) & $23,2(16)$ \\
\hline Correção de CIA (CCIA) & $2,9(2)$ \\
\hline Troca de Valva Aórtica (TVA) & $2,9(2)$ \\
\hline \multicolumn{2}{|l|}{ Tempo de Permanência na UTI } \\
\hline Até 2 dias & $50,7(35)$ \\
\hline$>2$ dias & $49,3(34)$ \\
\hline \multicolumn{2}{|l|}{ Tempo de CEC } \\
\hline Até 50 minutos & $26,1(18)$ \\
\hline 51-100 minutos & $68,1(47)$ \\
\hline Período >100 minutos & $5,8(4)$ \\
\hline \multicolumn{2}{|l|}{ Tempo de VM } \\
\hline Até 6 horas & $42(29)$ \\
\hline Período $>6$ horas & $58(40)$ \\
\hline
\end{tabular}

VMI: Ventilação Mecânica Invasiva.

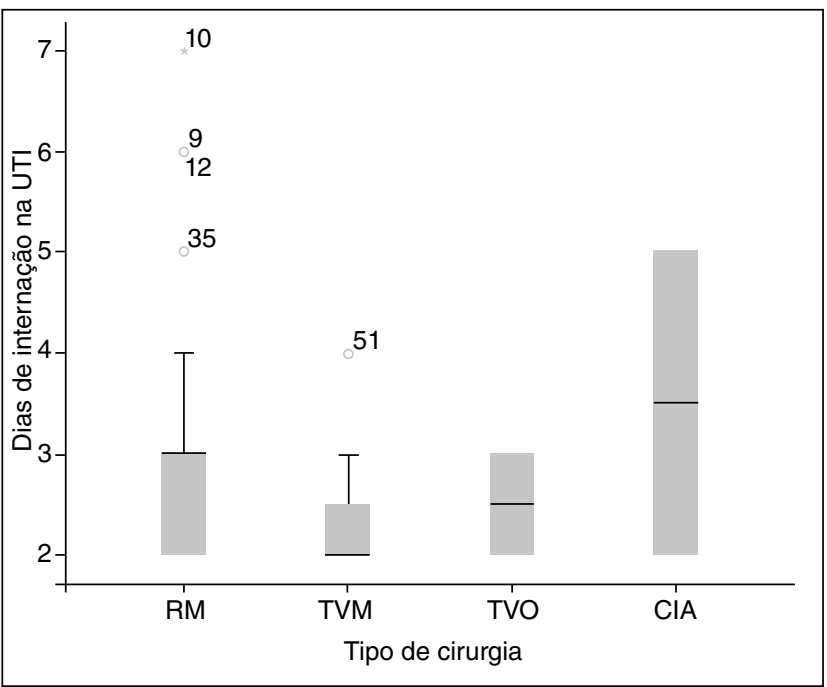

Figura 1: Relação entre o tempo de internação na Unidade de Terapia Intensiva e o tipo de cirurgia pulmonares pós-operatórias uma fonte significativa de mortalidade e morbidade. Visando reduzir essas complicações, é necessário identificar os pacientes em risco de complicações pulmonares pós-operatórias e otimizar a terapêutica ${ }^{2,8}$.

No estudo de Laizo et al. ${ }^{9}$, entre os 85 pacientes submetidos à cirurgia cardíaca, $51(60,00 \%)$ realizaram revascularização do miocárdio, $22(25,88 \%)$ trocaram de valva aórtica e 7 (8,23\%) trocaram de valva mitral. Dados semelhantes foram encontrados neste estudo, no qual a maioria realizou cirurgia de revascularização do miocárdio. Porém houve uma prevalência maior de pacientes submetidos à cirurgia de troca de valva mitral quando comparado com troca de valva aórtica. Em outro estudo ${ }^{10}$, com aproximadamente 30 pacientes, $50 \%$ fizeram uso da CEC e os outros 50\% não utilizaram a CEC para realização da cirurgia cardíaca. No presente estudo observou-se que $100 \%$ das cirurgias cardíacas foram com a utilização de CEC. Além disso, o tempo médio de CEC nessa pesquisa foi de $65,05 \pm 20,06$ minutos, praticamente o dobro do tempo encontrado no estudo de Guizillini et al. ${ }^{10}$, no qual há uma média de 109,10 $\pm 40,82$ minutos - o que representa uma diferença significativa quando comparados, ${ }^{10}$.

Em um estudo com 85 pacientes, o tempo de internação na UTI obteve uma média de 99,84 $\pm 90,24$ horas. Um paciente $(1,17 \%)$ permaneceu na UTI por 24 horas, $39(45,88 \%)$ por 48 horas, 12 $(14,11 \%)$ por 72 horas, $10(11,76 \%)$ por 96 horas, $9(10,58 \%)$ por 120 horas, e $14(16,47 \%)$ por mais de 120 horas $^{9}$.

A média de tempo de VMI na nesta pesquisa foi de 7,32 $\pm 2,66$ horas, enquanto Cockram et al. ${ }^{11}$ afirmaram que, dentre os 32 pacientes avaliados, todos foram extubados dentro de 18 horas de cirurgia - em média 12,40 $\pm 11,00$ horas —,com exceção de um indivíduo que necessitou de ventilação com pressão positiva intermitente por 68 horas devido a complicações pulmonares.

Em outro estudo, o tempo de ventilação mecânica maior que 12 horas foi um marcador de grande impacto na mortalidade, sendo essa uma de suas variáveis de predição de óbito hospitalar ${ }^{12}$.

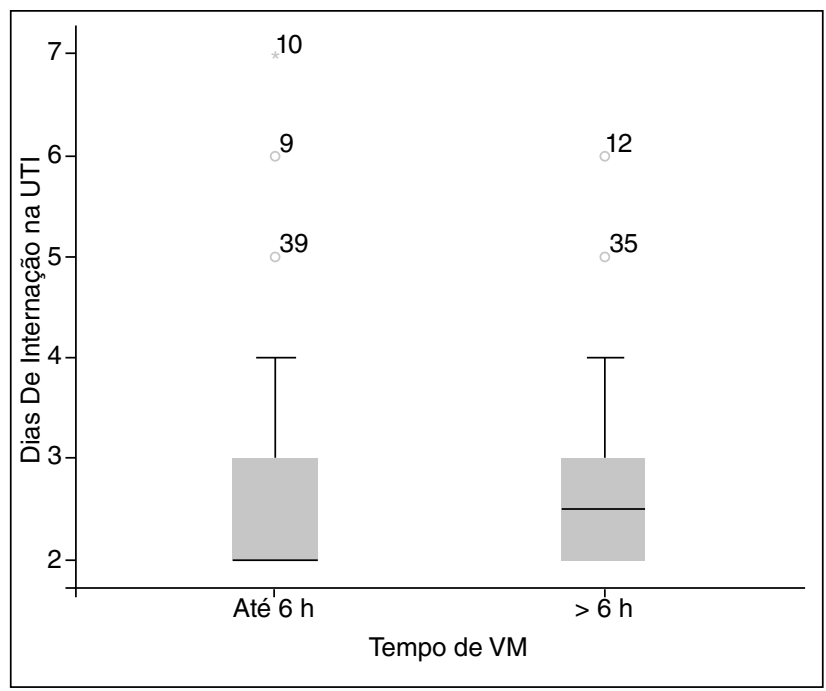

Figura 2: Relação entre o tempo de internação na Unidade de Terapia Intensiva e o tempo de Ventilação Mecânica Invasiva 
Em um levantamento sobre as características da VMI no Brasil, foi encontrado que a média de tempo de internamento em pacientes submetidos ao suporte ventilatório invasivo é de 16 dias, porém nesse trabalho foram utilizados variados perfis de pacientes ${ }^{13}$. Nozawa et al. ${ }^{14}$ afirmam que em pacientes submetidos à cirurgia cardíaca, que requerem VMI prolongada, os dados de mecânica respiratória e oxigenação não foram fatores envolvidos no sucesso ou insucesso no desmame. Porém, disfunção cardíaca e tempo de CEC influenciaram diretamente no sucesso do desmame. Piotto et al. ${ }^{15}$ realizaram um estudo dividindo os pacientes submetidos a cirurgia cardíaca em dois grupos, um com o tempo de VMI $>48$ horas e o outro com um tempo inferior a 48 horas, encontrando em ambos os grupos a idade, a doença pulmonar obstrutiva crônica e o tempo de CEC como os principais fatores para um tempo elevado de ventilação. Afirmou-se, ainda, que a descontinuação da VMI precoce esteja associada a melhores resultados.

No presente estudo, nenhum paciente permaneceu na VMI por um tempo superior a 48 horas. Já no estudo de Soares et al. ${ }^{16}$, $21,3 \%$ dos pacientes necessitaram de VMI por mais de 48 horas após a cirurgia. Foi relatado que esse aumento do tempo de VMI se deu devido a complicações pós-operatórias, principalmente associadas a alterações pulmonares.
Neste estudo encontrou-se um tempo médio de internamento na UTI pós-cirurgia cardíaca de $2,18 \pm 1,10$ dias. No trabalho de

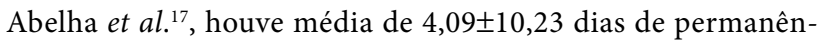
cia na UTI e de 25,51 $\pm 27,31$ dias de permanência hospitalar. Nesse mesmo trabalho, afirmou-se que o aumento do tempo de permanência superior a 3,5 dias está associado diretamente com a previsão de mortalidade, o que não foi verificado no presente estudo.

Em outro estudo, foram avaliados os resultados imediatos da cirurgia de revascularização miocárdica isolada em pacientes idosos; nesses trabalhos os autores observaram que o tempo médio de permanência na UTI foi de 5,7 dias. O tempo de internamento foi bastante superior ao da presente pesquisa, que apresentou apenas 2,1 dias de média ${ }^{18}$.

Uma limitação deste trabalho foi a não avaliação das comorbidades, variáveis pré-operatórias, complicações peri-operatórias e a falta de pacientes que permanecessem por um tempo superior a 24 horas na VMI, visto que esses fatores podem interferir no tempo de VMI e consequentemente no tempo de internação hospitalar.

Nesta pesquisa pode-se concluir que o tempo de ventilação mecânica invasiva não tem relação direta com o tempo de internamento na UTI. A provável justificativa para a falta de correlação entre as variáveis foi um tempo médio de VMI curto (7,2 horas), que não seria suficiente para provocar impacto na condição da internação.

\section{REFERÊNCIAS}

1. Brasil. Ministério da saúde. DATASUS. Informações de saúde: informações epidemiológicas e morbidade. Disponível em http:// www2.datasus.gov.br/DATASUS/index. php?area=02. Acesso em: 10 set. 2015

2. Herdy AH, Marcchi PL, Vila A, Tavares C, Collaço J, Niebauer $\mathrm{J}$, et al. Pre and postoperative cardiopulmonary rehabilitation in hospitalized patients undergoing coronary artery bypass surgery: a randomized controlled Trial. Am J Phys Med Rehabil. 2008:87(9):714-19. http://dx.doi.org/10.1097/PHM.0b013e3181839152

3. Butler R, Keenan SP, Inman KJ, Siibbald WJ, Block G. Is there a preferred technique for weaning the difficult to wean patient? A systematic review of the literature. Crit Care Med. 1999;27(11):2331-6

http://dx.doi.org/10.1097/00003246-199911000-00002

4. Kehlet H, Wilmore DW. Evidence-based surgical care and the evolution of fast-track surgery. Ann Surg. 2008;248(2):189-98. http://dx.doi.org/10.1097/SLA.0b013e31817f2c1a

5. Patel BK, Hall JB. Perioperative physiotherapy. Curr Opin Anesthesiol. 2013;26(2): 152-6. http://dx.doi.org/10.1097/ACO.0b013e32835e8b34

6. Nozawa E, Kobayashi E, Matsumoto ME, Feltrim MIZ, Carmona MJC, Auler Júnior JOC. Avaliação de fatores que influenciam no desmame de pacientes em ventilação mecânica prolongada após cirurgia cardíaca. Arq Bras Cardiol. 2003;80(3):301-5. http://dx.doi.org/10.1590/S0066-782X2003000300006

7. Gouvas N, Tan E, Windsor A, Xynos E, Tekkis PP. Fast-track vs standart care in colorectal surgery: a meta-analysis update. Int $\mathrm{J}$ Colorectal Dis. 2009;24(10):1119-31.

http://dx.doi.org/10.1007/s00384-009-0703-5
8. Leguisamo CP, Kalil RA, Furlani AP. A efetividade de uma proposta fisioterapêutica pré-operatória para cirurgia de revascularização do miocárdio. Rev Bras Cir Cardiovasc. 2005:20(2):134-41. http://dx.doi.org/10.1590/S0102-76382005000200008

9. Laizo A, Delgado FEF, Rocha GM. Complicações que aumentam o tempo de permanência na unidade de terapia intensiva na cirurgia cardíaca. Rev Bras Cir Cardiovasc. 2010;25(2):166-71. http://dx.doi.org/10.1590/S0102-76382010000200007

10. Guizillini S, Gomes WJ, Faresin SM, Carvalho ACC, Jaramillo JI, Alves FA, et al. Efeitos do local da inserção do dreno pleural na função pulmonar no pós-operatório de cirurgia de revascularização do miocárdio. Rev Bras Cir Cardiovasc. 2005;19(1):47-54 http://dx.doi.org/10.1590/S0102-76382004000100010

11. Cockram J, Jenkins S, Clugston R. Cardiovascular and respiratory responses to early ambulation and star climbing following coronary artery surgery. Physiot Theory Pract. 2009;15(1):3-15. http://dx.doi.org/10.1080/095939899307856

12. Gomes RV, Tura B, Tannus H, Mendonça Filho TF, Campos LAA Rouge $A$, et al. Rioescore: escore preditivo de mortalidade para pacientes submetidos à cirurgia cardíaca baseado em variáveis de pré, per e primeiro dia de pós-operatório. Rev SOCERJ. 2005;18(6):516-26.

13. Damasceno MPCD, David CMN, Souza PCSP, Chiavone PA, Cardoso LTQ, Amaral JLG, et al. Ventilação mecânica no Brasil: aspectos epidemiológicos. Rev Bras Ter Intensiva. 2006;18(3):219-28. http://dx.doi.org/10.1590/S0103-507X2006000300002

14. Nozawa E, Kobayashi E, Matsumoto ME, Feltrim MIZ, Carmona MJC, Auler Júnior JOC. Assessment of factors that influence weaning from long-term mechanical ventilation after; cardiac surgery. Arq Bras Cardiol. 2003;80(3):306-10. http://dx.doi.org/10.1590/S0066-782X2003000300006 
15. Piotto RF, Ferreira FB, Colósimo FC, Silva GS, Sousa AG, Braile DM. Fatores preditores independentes de ventilação mecânica prolongada em pacientes submetidos à cirurgia de revascularização miocárdica. Rev Bras Cir Cardiovasc. 2012;27(4):520-28. http://dx.doi.org/10.5935/1678-9741.20120093

16. Soares GMT, Ferreira DCS, Gonçalves MPC, Alves TGS, David FL, Henriques KMC et al. Prevalência das principais complicações pós-operatórias em cirurgias cardíacas. Rev Bras Cardiol. 2001;24(3):139-46
17. Abelha FJ, Castro MA, Landeiro NM, Neves AM, Santos CC. Mortalidade e o tempo de internação em uma unidade de terapia intensiva cirúrgica. Rev Bras Anestesiol. 2006;56(1):34-45. http://dx.doi.org/10.1590/S0034-70942006000100005

18. Oliveira ABF, Dias OM, Mello MM, Araújo S, Dragosavac D, Nucci $\mathrm{A}$, et al. Fatores associados à maior mortalidade e tempo de internação prolongado em uma unidade de terapia intensiva de adultos. Rev Bras Ter Intensiva. 2010;22(3):250-56. http://dx.doi.org/10.1590/S0103-507X2010000300006 